\title{
Non Gaussian multi-parameter Bayesian estimation through the mechanical equivalent of logical inference
}

\author{
Denise Bolognani $^{1,2}$, Andrea Verzobio ${ }^{2}$, Carlo Cappello ${ }^{2}$, Daniel Tonelli ${ }^{2}$, Branko \\ Glisic $^{1}$, Daniele Zonta ${ }^{3}$
}

\section{DOI: $\underline{\text { https://doi.org/10.5592/CO/BSHM2017.5.4 }}$}

\author{
${ }^{1}$ Princeton University, Princeton, NJ 08544 USA, \\ ${ }^{2}$ University of Trento, Mesiano Street, 77, Trento, 38123 Italy, \\ ${ }^{3}$ University of Strathclyde, Richmond Street, 16, Glasgow, G1 1XQ. Scotland, United Kingdom. \\ E-mails: 1,2db19@princeton.edu; ${ }^{2}$ andrea.verzobio8@gmail.com; ${ }^{2}$ carlo.cappello@unitn.it; \\ 2daniel.tonelli@unitn.it; ${ }^{1}$ bglisic@princeton.edu; ${ }^{3}$ daniele.zonta@strath.ac.uk
}

\begin{abstract}
Structural Health Monitoring requires engineers to understand the state of a structure from its observed response. When this information is uncertain, Bayesian probability theory provides a consistent framework for making inferences. However, structural engineers are often unenthusiastic about Bayesian logic, finding its application complicated and onerous, and prefer to make inference using heuristics. Here, we propose a quantitative method for logical inference based on a formal analogy between linear elastic mechanics and Bayesian inference with linear Gaussian variables. To start, we investigate the case of single parameter estimation, where the analogy is stated as follows: the value of the parameter is represented by the position of a cursor bar with one degree of freedom; uncertain pieces of information on the parameter are modelled as linear elastic springs in series or parallel, connected to the bar and each with stiffness equal to its accuracy; the posterior mean value and the accuracy of the parameter correspond respectively to the position of the bar in equilibrium and to the resulting stiffness of the mechanical system composed of the bar and the set of springs. Similarly, a multi-parameter estimation problem is reproduced by a mechanical system with as many degrees of freedom as the number of unknown parameters. In this case, the inverse covariance matrix of the parameters corresponds to the Hessian of the potential energy, while the posterior mean values of the parameters coincide with the equilibrium - or minimum potential energy - position of the mechanical system. We use the mechanical analogy to estimate, in the Bayesian sense, the drift of elongation of a bridge cable-stay undergoing continuous monitoring. We demonstrate how we can solve this in the same way as any other linear Bayesian inference problem, by simply expressing the potential energy of the equivalent mechanical system, with a few trivial algebraic steps and with the same methods of structural mechanics. We finally discuss the extension of the method to non-Gaussian estimation problems.
\end{abstract}

Keywords: decision making, value information, mechanical equivalent, Bayes' theorem, decision parameter estimation

\section{Introduction}

Structural engineers usually have a solid background in mechanics, yet not always a good relationship with probability theory. In most cases, this is not that critical because code-based design is practically probability-free, with serious probabilistic analysis typically being confined to the most recondite annexes of the codes (EN 1990:2002). It is different for those engineers who grapple with structural health monitoring (SHM), an activity where the objective is to estimate the state of a structure from an uncertain batch of observations provided by different kind of sensors, such as strain gauge (Zonta, Lanaro \& Zanon, 2003), fiber optic sensor (Inaudi \& Glisic, 2006) and accelerometers (Zonta, Wu, Pozzi et al., 2010). A consistent framework for making inferences from uncertain information is Bayesian probability theory (Sohn \& Law, 2000) (Bruschetta, Zonta, Cappello et al., 2013). Yet structural engineers are often unenthusiastic about Bayesian formal logic, finding its application complicated and burdensome, and they prefer to make inference by using heuristics. In this contribution, we wish to help structural engineers reconcile with probabilistic logic (Taynes, 2003) by 
suggesting a quantitative method for logical inference based on a formal analogy between mechanics and Bayesian probability. We will state the fundamentals of the analogy in the next section.

To start, we will limit the analogy to the case of linear Gaussian single-parameter estimation, which corresponds in the mechanical counterpart to mere linear elastic single-degree-of-freedom analysis: a cakewalk for structural engineers. In section 3, we apply this formal analogy to a classical inference problem: the estimation of the deformation of a cable belonging to a cable-stayed bridge, characterized by two independent parameters. We will carry out the simple problem of linear regression by solving the equivalent mechanical system of springs.

\section{Formulation of the analogy for a single parameter}

In this section, we refer to the problem of logical inference of a single parameter based on uncertain information (Cappello, Bolognani \& Zonta, 2015). The goal is to estimate a parameter $\theta$ based on a set of uncertain information $y_{i}$. Further assumptions are that all the uncertain quantities have Gaussian distribution, and that the relationship between information and parameter is linear. When the problem is linear and Gaussian, in principle we can solve any logical inference problem using the following two fundamental rules.

First inference rule or inverse-variance weighting rule (Ku, 1966). Given a set of $n$ observations $y_{i}$ of variance $\sigma_{i}^{2}$, the inverse of the variance $\sigma_{\theta}^{2}$ of the parameter is the sum of the inverse-variances of the observations, and the expected value of the parameter $\mu_{\theta}$ is the inverse-variance weighted sum of the observations:

$$
\frac{1}{\sigma_{\theta}^{2}}=\sum_{i=1}^{n} \frac{1}{\sigma_{i}^{2}}, \mu_{\theta}=\frac{\sum_{i=1}^{n} \frac{y_{i}}{\sigma_{i}^{2}}}{\sum_{i=1}^{n} \frac{1}{\sigma_{i}^{2}}} .
$$

Second inference rule or linear propagation of uncertainties (Rabinovich, 2005) (Kirkup \& Frenkel, 2006). The indirect measurement $y=x_{1}+\ldots+x_{m}$, being the sum of $m$ different arguments $x_{j}$ of variance $\sigma_{j}^{2}$, the variance of the observations is the sum of the variance of the arguments and the mean value of the indirect observation is the sum of the arguments:

$$
\sigma_{y}^{2}=\sum_{j=1}^{m} \sigma_{j}^{2}, \mu_{y}=\sum_{j=1}^{m} x_{j}
$$

Before proceeding it is also convenient, primarily to lighten notation, to introduce the quantity

$$
w=\sigma^{-2}=\frac{1}{\sigma^{2}} .
$$

The quantity $w$ is compatible with the official definition of accuracy (ISO5725-6:1994, 2012) and the word itself intuitively connects to the practical meaning of $\mathrm{w}$ : the higher the accuracy $\mathrm{w}$ of an observation is, the more accurate our knowledge about the parameter becomes. Therefore, in the rest of the paper we will refer to the inverse-variance w simply as accuracy. Based on that, we can reword and reformulate the two basic inference rules.

First inference rule. Given a set of $n$ observations $y_{i}$ with accuracy $w_{i}$, the accuracy $w_{\theta}$ of the parameter estimation is the sum of the accuracy of the observations, and the mean value of the parameter $\mu_{\theta}$ is the sum of the observations weighted with their accuracy:

$$
w_{\theta}=\sum_{i=1}^{n} w_{i}, \mu_{\theta}=\frac{\sum_{i=1}^{n} y_{i} w_{i}}{w_{\theta}} .
$$

Second inference rule. The indirect measurement $y=x_{1}+\ldots+x_{m}$ being the sum of $m$ different arguments $x_{j}$ of accuracy $w_{j}$, the inverse-accuracy of the observation is the sum of the inverse-accuracy of the arguments and the mean value of the indirect observation is the sum of the arguments: 


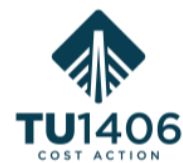

$$
\frac{1}{w_{y}}=\sum_{j=1}^{m} \frac{1}{w_{j}}, \mu_{y}=\sum_{j=1}^{m} x_{j}
$$

At this point, it is not difficult for a structural engineer to spot in (4a) the same form of the expression that provides the stiffness of a set of springs in parallel; and similarly, (5a) reminds of the stiffness expression of a set of springs in series. This opens a door to set an analogy between the world of logic and the world of mechanics. Particularly, the analogy statements (Cappello, Bolognani \& Zonta, 2015) are summarized in Table 1, while Figure 1 shows the mechanical representation of simple linear Gaussian inference problems.

(a)

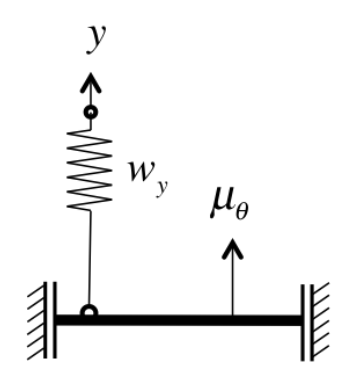

(b)

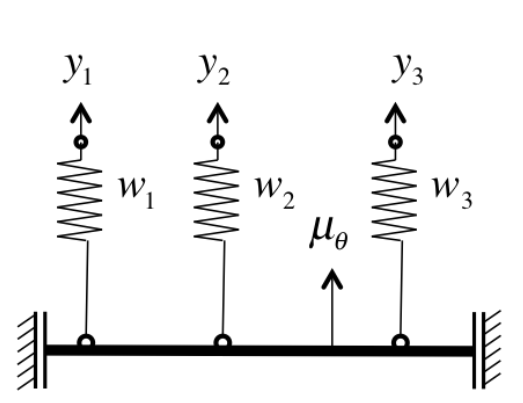

(c)

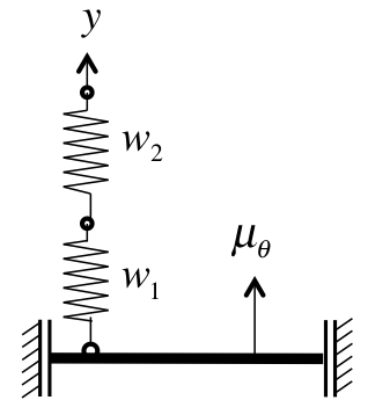

Fig. 1. Mechanical analogy of simple linear Gaussian inference problems: parameter estimation based on one observation (a), three uncorrelated observations (b), one observation affected by two uncorrelated sources of uncertainty (c).

Table 1. Analogy between inference and mechanical models

\begin{tabular}{ccc}
\hline Symbol & Logical meaning & $\begin{array}{c}\text { Mechanical } \\
\text { meaning }\end{array}$ \\
\hline$w, \sigma^{-2}$ & $\begin{array}{c}\text { accuracy, inverse- } \\
\text { variance }\end{array}$ & stiffness \\
\hline$\sigma^{2}$ & variance & flexibility \\
\hline$y$ & observation & pre-stretch \\
\hline$\mu$ & expected value & $\begin{array}{c}\text { equilibrium } \\
\text { displacement }\end{array}$ \\
\hline
\end{tabular}

\section{Extension of the analogy to $\mathrm{N}$ parameters}

Now, we analyse a generic inference problem with $\mathrm{N}$ unknown parameters to estimate, represented by the vector $\boldsymbol{\theta}=\left(\theta_{1}, \ldots ., \theta_{N}\right)^{\mathrm{T}}$. We imagine that each parameter is characterized by a prior mean value $\mu_{\theta_{i}}$ and a prior standard deviation $\sigma_{\theta_{i}}$; the latter is linked by the equation $w_{\theta_{i}}=\sigma_{\theta_{i}}^{-2}$ to the $\mathrm{i}^{\mathrm{th}}$ accuracy, which in our mechanical analogy represents the stiffness of the spring associated to each single parameter. The multivariate Gaussian distribution (Bishop, 2006), linked to the N-dimensional vector $\theta$, takes the form:

$$
\mathrm{N}(\boldsymbol{\mu}, \Sigma ; \theta)=\frac{1}{(2 \pi)^{\frac{N}{2}}} \frac{1}{|\Sigma|^{\frac{1}{2}}} \mathrm{e}^{\left\{-\frac{1}{2}(\theta-\mu)^{T} \Sigma^{-1}(\theta-\mu)\right\}},
$$

where $\mu$ is the $\mathrm{N}$-dimensional mean vector, containing the $\mathrm{N}$ values $\mu_{\theta_{i}}$ associated to each parameter, $\Sigma$ is the NxN covariance matrix, and $|\Sigma|$ denotes the determinant of $\Sigma$.

We can notice that the exponent is characterized by a quadratic form that corresponds to the potential energy $E_{p}(\theta)$ of a mechanical system with $\mathrm{N}$ degrees of freedom, related to the inference problem in question. It takes the following mathematical form: 


$$
\mathrm{E}_{\mathrm{p}}(\boldsymbol{\theta})=-\operatorname{In}(\mathrm{N}(\boldsymbol{\mu}, \Sigma ; \theta))=\frac{1}{2}(\mu-\theta)^{\mathrm{T}} \Sigma^{-1}(\mu-\theta)
$$

Here, we name the inverse of the covariance matrix $\Lambda=\Sigma^{-1}$; this is also known as accuracy matrix (Bishop, 2006). Its diagonal terms represent the posterior stiffness $w_{\theta_{i} \mid \mathbf{y}}$ of each single parameter $\theta_{i}$.

Now, to obtain the $\mathrm{N}$ diagonal elements to $\Lambda$ we must get the second derivative of $\mathrm{E}_{\mathrm{p}}(\boldsymbol{\theta})$ with respect to each of the parameters $\theta_{i}$; the elements out of diagonal are instead obtained by calculating the mixed derivatives of each parameter with respect to all other parameters. To obtain the covariance matrix we simply make the inverse of $\Lambda$. The diagonal elements of $\Sigma$ represent the posterior variance $\sigma_{\theta_{i} \mid \mathbf{y}}^{2}$ of each single parameter $\theta_{i}$. The posterior mean values $\mu_{\theta_{\mathrm{i}} \mathbf{y} \mathbf{y}}$ of each parameter $\theta_{i}$ correspond to those values that minimize the potential energy of our mechanical system. Therefore, to discover them, we have to resolve an algebraic system with $\mathrm{N}$ variables in which there are the partial derivatives of

$\mathrm{E}_{\mathrm{p}}(\boldsymbol{\theta})$, each with respect to each parameter $\theta_{i}$, set equal to zero.

\section{A case study: elongation of a cable belonging to Adige Bridge}

Structural monitoring has been recognized as a powerful information tool, especially with regard to bridges management (Pozzi, Zonta, Wang et al., 2010), and request a deep knowledge of Bayesian rules. For this reason, we apply our method to the Adige Bridge (Cappello, Zonta, Pozzi et al., 2015) (Bruschetta, Zonta, Cappello et al., 2013), a two-span cable-stayed bridge located ten kilometres north of the city of Trento, Italy (Figure 2). The composite deck is made from 4 "I"-section steel girders and a $25 \mathrm{~cm}$ cast-on-site concrete slab. The deck is also supported by 12 stay cables, 6 on each side, which have a diameter of $116 \mathrm{~mm}$ and $128 \mathrm{~mm}$. Their operational design load varies from 5,000 kN to 8,000 $\mathrm{kN}$. The cables are anchored to the bridge tower, consisting of four pylons and located in the middle of the bridge. When the construction was completed, the Italian Autonomous Province of Trento, which owns and manages the bridge, decided to install a monitoring system to continuously record force and elongation of the stay cables. Elongations are recorded by $1 \mathrm{~m}$ long gauge sensors, placed on each of the 12 cables. These fiber-optical sensors (FOS) (Glisic, Posenato \& Inaudi, 2007) are based on fiber Bragg gratings (FBG) which rely on a principle similar to that of photonic crystals (Zonta, Chappini, Chiasera et al., 2009) but provide better precision. These sensors also record local temperature for thermal compensation.
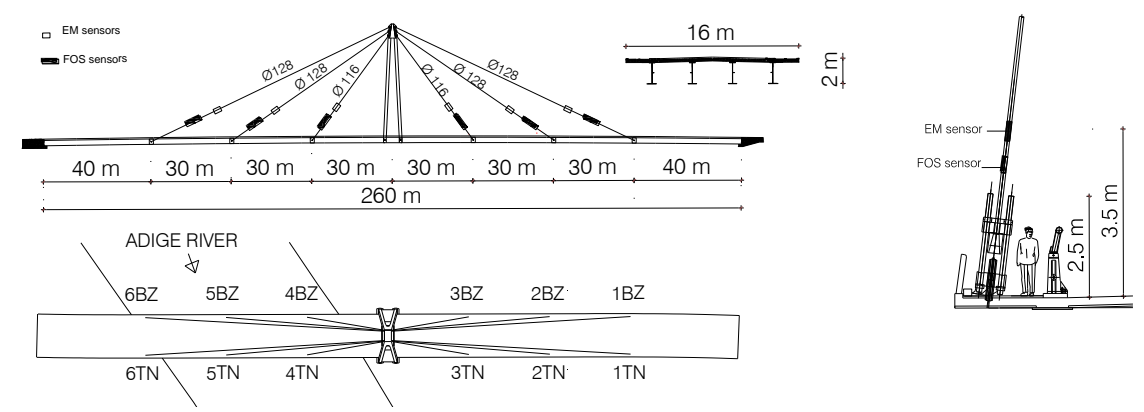

Fig. 2. Longitudinal section of the bridge and sensor layout (upper left); plan view of the bridge (lower left); cross-section of the bridge (right).

\subsection{Two parameters to estimate}

As an example, we use data acquired from October 12, 2011, to November 25, 2012, for cable 1TN, purified of the effect of temperature. We consider only one sample a day, recorded between 4 AM and $6 \mathrm{AM}$, as we assume the temperature in this period to be constant. We have discarded those days in which no samples were found in the time interval. Figure 3 shows the data acquired, expressed in terms of difference of deformation and time:

$$
\Delta y=y_{i}-y_{1}, \quad \Delta t=t_{i}-t_{1} .
$$



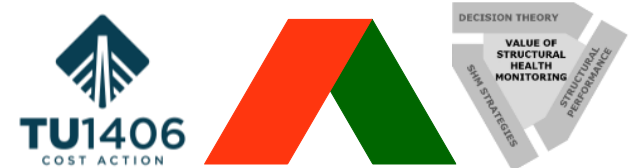

The Value of Structural Health Monitoring for the reliable Bridge Management

Zagreb 2-3 March 2017

During the analysis, 411 deformation measurements were recorded with an uncertainty for each measurement equal to $w_{\mathrm{y}}=0.0016 \mu \varepsilon^{-2}$, i.e. $\sigma_{\mathrm{y}}=25 \mu \varepsilon$. This is clearly a classical problem of linear regression. We have to estimate the two parameters that best characterize the straight line fitting our time-dependent data set. The function employed is:

$$
y=y_{0}+\varphi \cdot t,
$$

where $\mathrm{y}_{0}$ is the intercept and $\varphi$ the slope of the straight line fitting our dataset. As we said before, the goal is to estimate the vector of the parameters $\theta=\left(y_{0}, \varphi\right)^{\mathrm{T}}$ that characterizes the parametric model resulting in the observations $\mathbf{y}=\left(\mathrm{y}_{1}, \mathrm{y}_{2}, \ldots ., \mathrm{y}_{\mathrm{N}}\right)^{\mathrm{T}}$, linearly dependent on the time $t$, as shown in Figure 4 . We can represent the problem as a bar with two degrees of freedom: vertical translation and rotation. According to the parametric model defined in (9), we consider the slope of the bar linked to the parameter $\varphi$, its length to the time $t$ and its distance from the ground floor to the parameter $y_{0}$.

Based on our experience, we assign to the two parameters $\varphi$ and $t$ two prior Gaussian distributions that give us the initial information about the state of the bar. We connect the left-hand end of the rigid bar to a vertical linear elastic spring with flexibility equal to the standard deviation of the prior distribution associated to the parameter $y_{0}$ and pre-stretch equal to its mean value. We connect the same end to a torsion spring with flexibility and imposed rotation equal respectively to the standard deviation and the mean value of the prior distribution associated to the parameter $\varphi$, as shown in Figure 4. Finally, we introduce the measurements as a system of linear springs, each with flexibility and pre-stretch equal respectively to the standard deviation and value associated to a single measurement. Each spring is placed at a distance from the torsion spring equal to the corresponding interval of time $t_{\mathrm{i}}$. The elastic potential of the mechanical system of Figure 4 becomes:

$$
\mathrm{E}_{p}\left(y_{0}, \varphi\right)=\frac{1}{2} w_{y}\left(y_{0}-\mu_{y_{0}}\right)^{2}+\frac{1}{2} w_{\varphi}\left(\varphi-\mu_{\varphi}\right)^{2}+\frac{1}{2} w_{y} \sum_{i=1}^{N}\left[\left(y_{0}+\varphi t_{i}\right)-y_{i}\right]^{2},
$$

where $\Delta y_{i}=y_{0}+\varphi \cdot t_{i}-y_{i}$ represents the elongation suffered by the $N$ springs linked to the observations, due to a generic translation $y_{0}$ and a generic rotation $\varphi$ imposed on the system. The accuracy matrix is simply the Hessian matrix of (10):

$$
\boldsymbol{\Lambda}=\left[\begin{array}{ll}
\frac{\delta^{2} \mathrm{E}_{\mathrm{p}}\left(y_{0}, \varphi\right)}{\delta y_{0}{ }^{2}} & \frac{\delta^{2} \mathrm{E}_{\mathrm{p}}\left(y_{0}, \varphi\right)}{\delta y_{0} \delta \varphi} \\
\frac{\delta^{2} \mathrm{E}_{\mathrm{p}}\left(y_{0}, \varphi\right)}{\delta \varphi \delta y_{0}} & \frac{\delta^{2} \mathrm{E}_{\mathrm{p}}\left(y_{0}, \varphi\right)}{\delta \varphi^{2}}
\end{array}\right] .
$$

The inverse of the matrix (11) represents the covariance matrix $\Sigma$ : the first term of its diagonal is the posterior variance associated to the parameter $y_{0}$ while the second term on the same diagonal is the posterior variance associated to the parameter $\varphi$. To identify instead the values $\mu_{y_{0} \mid y}$ and $\mu_{\varphi \mid y}$ that represent the posterior mean values associated respectively to the parameters $y_{0}$ and $\varphi$, we must solve the system formed by the first derivative of (10) with respect to the parameter $y_{0}$ and the parameter $\varphi$, set equal to zero.

$$
\left\{\begin{array}{l}
\frac{\partial \mathrm{E}_{\mathrm{p}}(\boldsymbol{\theta})}{\partial y_{0}}=w_{y_{0}}\left(y_{0}-\mu_{y_{0}}\right)+w_{y} \sum_{i=1}^{N}\left[\left(y_{0}+\varphi t_{i}\right)-y_{i}\right]=0 \\
\frac{\partial \mathrm{E}_{\mathrm{p}}(\boldsymbol{\theta})}{\partial \varphi}=w_{\varphi}\left(\varphi-\mu_{\varphi}\right)+w_{y} \sum_{i=1}^{N} t_{i}\left[\left(y_{0}+\varphi \mathrm{t}_{i}\right)-y_{i}\right]=0
\end{array} .\right.
$$

The solutions of the system (12) give us the values of $\mu_{y_{0} \mid \mathrm{y}}$ and $\mu_{\varphi \mid \mathrm{y}}$, that represent the posterior mean values associated respectively to the parameters $y_{0}$ and $\varphi$ and that minimize the potential $\mathrm{E}_{\mathrm{p}}\left(y_{0}, \varphi\right)$ of our mechanical system. Now we can substitute the numerical values into the equations formulated above, and we obtain the final outcomes reported in Table 2, compared with the prior values of the parameters. Figure 3 reports the two straight lines interpolating our dataset. We obtain the same results 
as applying the flexibility method to the same mechanical system (Cappello, Bolognani \& Zonta, 2015), although, with the potential energy, we considerably reduce the computational cost of the algebra.

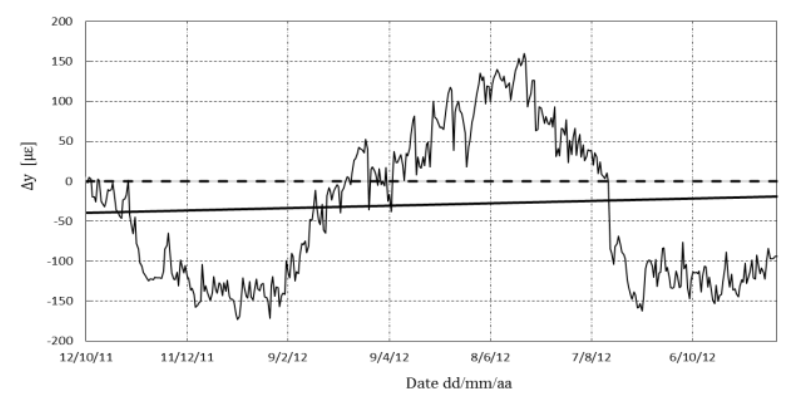

Fig. 3. Relative strain of cable $1 \mathrm{TN}$ and interpolating lines.

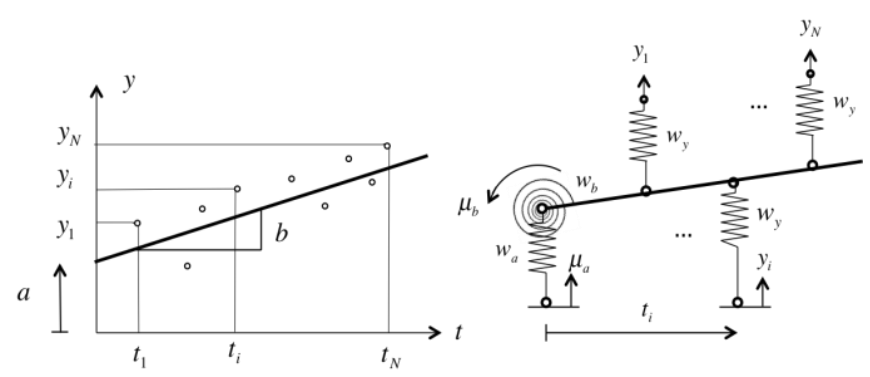

Fig. 4. Linear regression problem in the world of Mechanics.

Table 2. Prior and posterior values of the parameters to estimate

\begin{tabular}{cccc}
\hline \multicolumn{5}{c}{ Prior distributions } \\
\hline Parameter $y_{0}$ & \multicolumn{3}{c}{ Parameter $\varphi$} \\
\hline$w_{y_{0}}\left[\mu \varepsilon^{-2}\right]$ & 0.0025 & $w_{\varphi}\left[\mu \varepsilon^{-2} d a y^{2}\right]$ & 1 \\
\hline$\sigma_{y_{0}}[\mu \varepsilon]$ & 20.00 & $\sigma_{\varphi}\left[\mu \varepsilon d a y^{-1}\right]$ & 1.0000 \\
\hline$\mu_{y_{0}}[\mu \varepsilon]$ & 0.00 & $\mu_{\varphi}\left[\mu \varepsilon d a y^{-1}\right]$ & 0.0000 \\
\hline \multicolumn{5}{c}{ Posterior distributions } \\
\hline Parameter $y_{0}$ & \multicolumn{4}{c}{ Parameter $\varphi$} \\
\hline$w_{y_{0}}\left[\mu \varepsilon^{-2}\right]$ & 0.6601 & $w_{\varphi}\left[\mu \varepsilon^{-2} d a y^{2}\right]$ & 36893 \\
\hline$\sigma_{y_{0}}[\mu \varepsilon]$ & 2.44 & $\sigma_{\varphi}\left[\mu \varepsilon d a y^{-1}\right]$ & 0.0103 \\
\hline$\mu_{y_{0}}[\mu \varepsilon]$ & -49.07 & $\mu_{\varphi}\left[\mu \varepsilon d a y^{-1}\right]$ & 0.0473
\end{tabular}

\subsection{Three parameters to estimate}

We now extend the case of Adige Bridge, presented in the previous Section, by introducing the effect of temperature $\Delta \hat{T}$. Thus, we must estimate an additional parameter $\alpha$ and the model that fits our time dependent dataset becomes the following:

$$
\Delta \hat{y}=y_{0}+\alpha \cdot \Delta \hat{T}+\varphi \cdot \Delta \hat{t}
$$

In Figure 5, we can note the $N$ translation springs linked to the different measurements with stiffness $w_{L H}=\sigma_{L H}{ }^{-2}=0.0016 \mu \varepsilon^{-2}$ and the springs linked to the prior distribution: a translation spring associated to the parameter $y_{0}$, a rotational spring associated to $\alpha$ and a rotational spring associated to $\varphi$, whose numerical values are the same as the case in the previous Section. To determine the posterior standard deviation of the three parameters to estimate $\left(\mathrm{y}_{0}, \alpha, \varphi\right)$ we have to express the potential energy $\mathrm{E}_{\mathrm{p}}\left(y_{0}, \alpha, \varphi\right)$ of the mechanical system represented in Figure 5, as a function of the three 

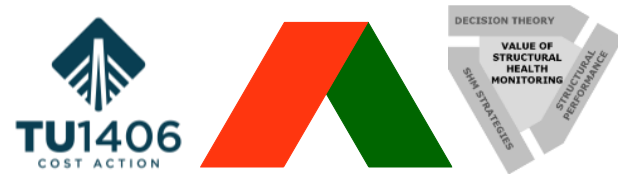

The Value of Structural Health Monitoring for the reliable Bridge Management

Zagreb 2-3 March 2017

unknown parameters. We can now obtain the accuracy matrix $\Lambda$ simply by calculating the Hessian Matrix associated to $\mathrm{E}_{\mathrm{p}}\left(y_{0}, \alpha, \varphi\right)$, and the covariance matrix from the inverse of $\Lambda$.

To discover the values $\mu_{y_{0} \mid y}, \mu_{\alpha \mid y}$ and $\mu_{\varphi \mid y}$, which represent the posterior mean values associated respectively to the parameters $y_{o}, \alpha$ and $\varphi$, we must solve the system formed by the first derivative of the potential energy with respect to the three parameters, set equal to zero. Figure 6 shows the graphical representation, using the software Matlab, of the two surfaces fitting our data set. Finally, Table 3 reports the numerical values obtained from the posterior distribution of the parameters.
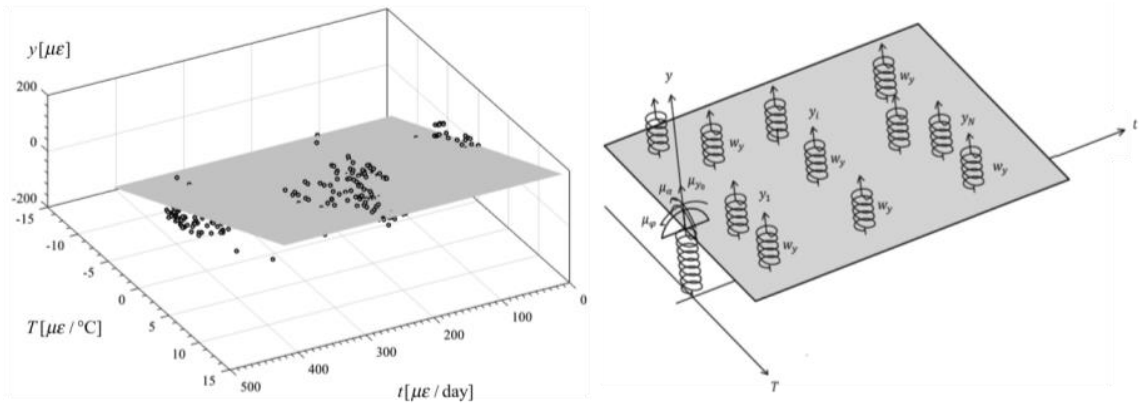

Fig. 5. Representation of a linear regression problem with three parameters to estimate, in the world of Mechanics.

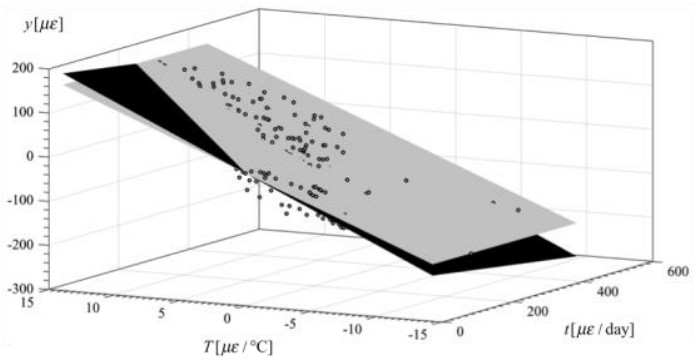

Fig. 6. Representation of the two fitting surfaces associated to the prior parameters (grey) and to the posterior parameters (black).

Table 3. Posterior values of the three parameters to estimate

\begin{tabular}{cccccc}
\hline \multicolumn{5}{c}{ Posterior distributions } \\
\hline \multicolumn{2}{c}{ Parameter $y_{0}$} & \multicolumn{2}{c}{ Parameter $\varphi$} & \multicolumn{2}{c}{ Parameter $\alpha$} \\
\hline$w_{y_{0}}\left[\mu \varepsilon^{-2}\right]$ & 0.6601 & $w_{\varphi}\left[\mu \varepsilon^{-2} d a y^{2}\right]$ & 36893 & $w_{\alpha}\left[\mu \varepsilon^{-2}{ }^{\circ} C^{2}\right]$ & 27.88 \\
\hline$\sigma_{y_{0}}[\mu \varepsilon]$ & 2.54 & $\sigma_{\varphi}\left[\mu \varepsilon d a y^{-1}\right]$ & 0.0106 & $\sigma_{\alpha}\left[\mu \varepsilon^{\circ} C^{-1}\right]$ & 0.20 \\
\hline$\mu_{y_{0}}[\mu \varepsilon]$ & 0.48 & $\mu_{\varphi}\left[\mu \varepsilon d a y^{-1}\right]$ & -0.1209 & $\mu_{\alpha}\left[\mu \varepsilon^{\circ} C^{-1}\right]$ & 13.80 \\
\hline
\end{tabular}

\section{Non Gaussian variables: single parameter estimation}

How does theory of the mechanical equivalent change if we decide to involve non-Gaussian variable? As it is logical, we will obtain non-linear springs, whose constitutive laws vary according to the probability distributions that characterize them. To extend the mechanical analogy to distribution other than the Gaussian one is very simple thanks to the three basic rules that will presented in section 5.2.

\subsection{The Gaussian case}

To better understand how to proceed, we must first start to work with a Gaussian distribution. We consider a univariate Gaussian distribution with deviation standard $\sigma_{x}$ and mean value $\mu_{x}$, where $x$ 
represents the unknown parameter to estimate. As we obtained in Section 3, the elastic potential associated to this is:

$$
\mathrm{N}(\mu, \sigma ; x)=\frac{1}{x \sqrt{2 \pi}} \mathrm{e}^{-\frac{1}{2 \sigma^{2}}(x-\mu)^{2}}, \mathrm{E}_{\mathrm{p}}(x)=\frac{1}{2} w(x-\mu)^{2} .
$$

We calculate now the natural logarithm of the probability function defined before:

$$
\operatorname{In}(\mathrm{N}(\mu, \sigma ; x))=\operatorname{In}\left(\frac{1}{\sigma \sqrt{2 \pi}}\right)+\operatorname{In}\left(\mathrm{e}^{-\frac{1}{2 \sigma^{2}}(x-\mu)^{2}}\right)=-\frac{1}{2 \sigma^{2}}(x-\mu)^{2}+a,
$$

where $a$ is an additive constant, which we can omit.

By focusing our attention on (15), it is not so difficult discover that the relation between the Gaussian distribution and the elastic potential is the following:

$$
\mathrm{E}_{\mathrm{p}}(x)=-\operatorname{In}(\mathrm{N}(\mu, \sigma ; x))=\frac{1}{2 \sigma^{2}}(x-\mu)^{2} .
$$

As we already know, we can obtain the stiffness of the spring $w$ that characterizes the Gaussian distribution in this way:

$$
\mathrm{k}(x)=\frac{\mathrm{d}^{2} \mathrm{E}_{\mathrm{p}}(x)}{\mathrm{d} x^{2}}=\frac{1}{\sigma^{2}}=w,
$$

and, according to the laws of the Mechanics (Ward, 1992), the elastic force F, i.e. the return force, which correlates the stiffness of the spring with its pre-stretch, is conservative. This means that we can define a scalar potential (potential energy) that the force will be the gradient or, in the case of a dimension, the first derivative.

$$
\mathrm{F}(x)=\frac{\mathrm{dE}_{\mathrm{p}}(x)}{\mathrm{d} x}=\mathrm{k}(x) \cdot x .
$$

With these premises, it is clear that a Gaussian distribution, in the mechanical equivalent analogy is represented as a spring with a parabolic potential, i.e. proportional to the square of $x$, and with linear constitutive law, expressed by the relation with the return force $\mathrm{F}$ and the parameter $x$. It is evident that, in the case of a Gaussian distribution, the relation between the elastic force and the unknown parameter, which represents the pre-stretch of the spring in the mechanical analogy, is linear: this is the reason why, in the preceding sections, we have schematized inference problems as mechanical systems composed by linear elastic springs in series or in parallel. The mechanical properties linked to a Gaussian distribution with the statistical quantities reported in Table 4, are shown in Table 19.

Table 4. Statistical properties of the probability distribution $\mathrm{N}(1,1 ; x)$

\begin{tabular}{ll}
\hline $\boldsymbol{\mu}$ & 1 \\
\hline $\boldsymbol{\sigma}$ & 1 \\
\hline mean & $\mu=1$ \\
\hline variance & $\sigma^{2}=1$ \\
\hline median & $\mu=1$ \\
\hline mode & $\mu=1$ \\
\hline
\end{tabular}

\subsection{Non-linear cases}

Now, we denote with $f(x ; a, b)$ a generic probability distribution, where $x$ is the unknown parameter to estimate, $a$ and $b$ the parameters that characterize the probability distribution under exam. According 

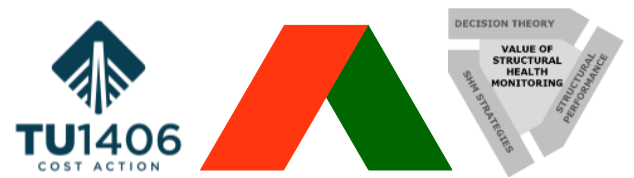

The Value of Structural Health Monitoring for the reliable Bridge Management

Zagreb 2-3 March 2017

to Eq. (16-18), the potential energy, the elastic force and the stiffness linked to the spring representing the generic distribution $f$ are the following:

$$
\mathrm{E}_{p}(x)=-\operatorname{In}(f), \mathrm{F}(x)=\frac{\partial \mathrm{E}_{p}(x)}{\partial x}, k(x)=\frac{\partial^{2} \mathrm{E}_{p}(x)}{\partial^{2} x} .
$$

In the following sections we will report some examples, regarding the main probability distributions used in the world of logic, and we will try to define for each the mechanical features of the springs that represent them.

\subsubsection{Lognormal distribution}

The lognormal distribution (Forbes et al., 2011) is applicable to random variables that are constrained by zero but have a few very large values. The resulting distribution is asymmetrical and positively skewed. In particular, in engineering field, the lognormal distribution is often used to describe the fatigue behaviour of many mechanical components and the mechanical resistance of structural materials, as the steel.

The application of a logarithmic transformation to the data can allow the data to be approximated by the symmetrical normal distribution, although the absence of negative values may limit the validity of this procedure. In other words, it is the probability distribution of a random variable $x$ whose logarithm $\operatorname{In}(x)$ follows a normal distribution, and it takes the following form:

$$
\mathrm{l}(\lambda, \varepsilon ; x)=\frac{1}{x \varepsilon \sqrt{2 \pi}} \mathrm{e}^{-\frac{1}{2 \varepsilon^{2}}(\operatorname{In}(x)-\lambda)^{2}} \text { with } 0<x<+\infty,
$$

where $\lambda$ is the mean of $\operatorname{In}(x)$ and $\varepsilon$ the standard deviation of $\operatorname{In}(x)$, which are both dimensionless. Table 5 shows the statistical properties of the lognormal distribution.

Table 5. Statistical properties of the probability distribution $1(\lambda, \varepsilon ; x)$

\begin{tabular}{ll}
\hline median & $e^{\lambda}$ \\
\hline mean & $e^{\lambda+\frac{1}{2} \varepsilon^{2}}$ \\
\hline variance & $e^{2 \lambda+\varepsilon^{2}} \cdot\left(e^{\varepsilon^{2}}-1\right)$ \\
\hline mode & $e^{\lambda-\varepsilon^{2}}$ \\
\hline
\end{tabular}

How can we model a spring representing $1(\lambda, \varepsilon ; x)$ ? The answer is simple: we must use the three aforementioned expressions $(19 a, b, c)$, to obtain the trend of the potential, of the elastic force and of the stiffness of the spring linked to the lognormal distribution.

$$
\mathrm{E}_{p}(x)=-\operatorname{In}(\mathrm{l}(\lambda, \varepsilon ; x))=\frac{1}{2 \varepsilon^{2}}(\operatorname{In}(x)-\lambda)^{2}+\operatorname{In}(x)+a,
$$

where $a$ is an additive constant that we can neglect.

$$
\begin{gathered}
\mathrm{F}(x)=\frac{\partial \mathrm{E}_{p}(x)}{\partial x}=\frac{1}{x \varepsilon^{2}}\left(\operatorname{In}(x)-\lambda+\varepsilon^{2}\right), \\
k(x)=\frac{\partial^{2} \mathrm{E}_{p}(x)}{\partial^{2} x}=\frac{1}{x^{2} \varepsilon^{2}}\left(1-\operatorname{In}(x)+\lambda-\varepsilon^{2}\right) .
\end{gathered}
$$

The mechanical properties linked to a lognormal distribution with the statistical quantities reported in Table 6, are shown in Table 19. 
Table 6. Statistical properties of the probability distribution $1(1,1 ; x)$

\begin{tabular}{ll}
\hline$\lambda$ & 1 \\
\hline$\varepsilon$ & 1 \\
\hline median & $e^{\lambda} \simeq 2.72$ \\
\hline mean & $e^{\lambda+\frac{1}{2} \varepsilon^{2}} \simeq 4.48$ \\
\hline variance & $e^{2 \lambda+\varepsilon^{2}} \cdot\left(e^{\varepsilon^{2}}-1\right) \simeq 34.51$ \\
\hline mode & $e^{\lambda-\varepsilon^{2}}=1$ \\
\hline
\end{tabular}

\subsubsection{Extreme value (Gumbel) distribution}

The extreme value distribution (Gao \& Sun) was developed as the distribution of the largest of a number of values and was originally applied to the estimation of flood levels. It has since been applied to the estimation of the magnitude of earthquakes. The distribution may also be applied to the study of athletic and other records. We consider the distribution of the largest extreme. Reversal of the sign of $x$ gives the distribution of the smallest extreme. This is the Type I, the most common of three extreme value distributions, known as the Gumbel distribution. Its probability density function takes the following form:

$$
\mathrm{g}(a, b ; x)=\frac{1}{b} e^{\frac{-(x-a)}{b}-e^{\frac{-(x-a)}{b}}} \text { with }-\infty<x<+\infty,
$$

where $a$ and $b$ are the parameters that characterize the distribution.

The main statistic quantities of the Gumbel distribution are reported in Table 7.

Table 7. Main statistical quantities of a random variable $x$, belonging to a Gumbel distribution

\begin{tabular}{ll}
\hline median & $a-b \cdot \operatorname{In}(\operatorname{In}(2))$ \\
\hline mean & $a-b \cdot \Gamma^{\prime}(1)$ \\
\hline variance & $b^{2} \pi^{2} / 6$ \\
\hline mode & $a$ \\
\hline
\end{tabular}

where $\Gamma^{\prime}(1)=-0.57722$ is the first derivative of the Gamma function $\Gamma(n)$ with respect to $n=1$.

Extreme value variates correspond to the limit, as $n$ tends to infinity, of the maximum value of $n$ independent random variates with the same continuous distribution. Logarithmic transformations of extreme value variates of Type II (Frèchet) and Type III (Weibull) correspond to Type I Gumbel variates. After introducing the main properties of the distribution, we are able, with the same Eq. $(19 \mathrm{a}, \mathrm{b}, \mathrm{c})$ of the previous Section, to spot the potential, the elastic force and the stiffness function linked to a Gumbel distribution $\mathrm{g}(a, b ; x)$.

$$
\mathrm{E}_{\mathrm{p}}(x)=-\operatorname{In}(\mathrm{g}(a, b ; x))=\operatorname{In}(b)+\frac{(x-a)}{b}+e^{\frac{-(x-a)}{b}}=\frac{(x-a)}{b}+e^{\frac{-(x-a)}{b}}+c,
$$

where $c$ is an additive constant that we can neglect.

$$
\begin{gathered}
\mathrm{F}(x)=\frac{\partial \mathrm{E}_{\mathrm{p}}(x)}{\partial x}=\frac{1}{b}-\frac{1}{b} e^{\frac{-(x-a)}{b}}=\frac{1}{b}\left(1-e^{\frac{-(x-a)}{b}}\right), \\
\mathrm{k}(x)=\frac{\partial^{2} \mathrm{E}_{\mathrm{p}}(x)}{\partial x^{2}}=\frac{1}{b^{2}} e^{\frac{-(x-a)}{b}} .
\end{gathered}
$$



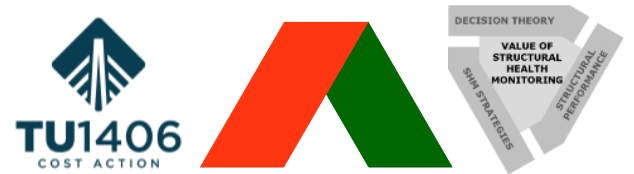

The Value of Structural Health Monitoring for the reliable Bridge Management

Zagreb 2-3 March 2017

The mechanical properties linked to a lognormal distribution with the statistical quantities reported in Table 8, are shown in Table 19.

Table 8. Statistical properties of the probability distribution $\mathrm{g}(1,1 ; x)$

\begin{tabular}{ll}
\hline $\mathbf{a}$ & 1 \\
\hline $\mathbf{b}$ & 1 \\
\hline median & $a-b \cdot \ln (\ln (2)) \simeq 1.37$ \\
\hline mean & $a-b \cdot \Gamma^{\prime}(1) \simeq 1.58$ \\
\hline variance & $b^{2} \pi^{2} / 6 \simeq 1.64$ \\
\hline mode & $a=1$ \\
\hline
\end{tabular}

\subsubsection{Cauchy distribution}

The Cauchy distribution (Forbes et al., 2011) is of mathematical interest due to the absence of defined moments. Its probability density function takes the following form:

$$
c(a, b ; x)=\left\{\pi b\left[1+\left(\frac{x-a}{b}\right)^{2}\right]\right\}^{-1} \text { with }-\infty<x<+\infty,
$$

where $a$ and $b$ are the parameters that characterize the distribution. The main statistic quantities of the Cauchy distribution are reported in Table 9.

Table 9. Main statistical quantities of a random variable $\mathrm{x}$, belonging to a Cauchy distribution

\begin{tabular}{ll}
\hline median & $a$ \\
\hline mean & Does not exist \\
\hline variance & Does not exist \\
\hline mode & $a$ \\
\hline
\end{tabular}

The Cauchy distribution is unimodal and symmetric, with much heavier tails than the normal. The probability density function is symmetric about a, with upper and lower quartiles, $a \pm b$. The potential, the elastic force and the stiffness function linked to the Gumbel distribution $\mathrm{c}(a, b ; x)$ are:

$$
\mathrm{E}_{\mathrm{p}}(x)=-\mathrm{In}\left(\left\{\pi b\left[1+\left(\frac{x-a}{b}\right)^{2}\right]\right\}^{-1}\right)=\operatorname{In}\left(1+\left(\frac{x-a}{b}\right)^{2}\right)+c,
$$

where $\mathrm{c}$ is an additive constant that we can neglect.

$$
\begin{gathered}
\mathrm{F}(x)=\frac{\partial \mathrm{E}_{\mathrm{p}}(x)}{\partial x}=\frac{1}{1+\left(\frac{x-a}{b}\right)^{2}} \cdot \frac{2}{b}\left(\frac{x-a}{b}\right)=\frac{2(x-a)}{b^{2}+(x-a)^{2}}, \\
\mathrm{k}(x)=\frac{\partial^{2} \mathrm{E}_{\mathrm{p}}(x)}{\partial x^{2}}=\frac{2(x-a)-2}{\left(b^{2}+(x-a)^{2}\right)^{2}} .
\end{gathered}
$$

The mechanical properties linked to a lognormal distribution with the statistical quantities reported in Table 10, are shown in Table 19. 
Table 10. Statistical properties of the probability distribution $\mathrm{c}(1,1 ; x)$

\begin{tabular}{cc}
\hline $\mathbf{a}$ & 1 \\
\hline $\mathbf{b}$ & 1 \\
\hline median & $a=1$ \\
\hline mean & Do not exist \\
\hline variance & Do not exist \\
\hline mode & 1 \\
\hline
\end{tabular}

\subsubsection{Beta distribution}

Applications include modeling random variables that have a finite range, $a$ to $b$. An example is the distribution of activity times in project networks. The beta distribution is frequently used as a prior distribution for binomial proportions in Bayesian analysis. Its probability density function takes the following form (Forbes et al., 2011):

$$
\beta(v, \omega ; x)=\frac{x^{v-1}(1-x)^{\omega-1}}{\mathrm{~B}(v, \omega)} \text { with } 0 \leq x \leq 1,
$$

where $v>0 a$ and $\omega>0$ are the parameters that ${ }_{1}$ characterize the distribution, and $\mathrm{B}(v, \omega)$ is the Beta function with arguments $v, \omega$ given by $\mathrm{B}(v, \omega)=\int_{0} u^{\nu-1}(1-u)^{\omega-1} d u$.

The main statistic quantities of the Cauchy distribution are reported in Table 11

Table 11. Main statistical quantities of a random variable $\mathrm{x}$, belonging to a Beta distribution

\begin{tabular}{cc}
\hline mean & $v /(v+\omega)$ \\
\hline variance & $v \omega /\left[(v+\omega)^{2}(v+\omega+1)\right]$ \\
\hline mode & $(v-1) /(v+\omega-2), v>1, \omega>1$ \\
\hline
\end{tabular}

The potential, the elastic force and the stiffness function linked to the Gumbel distribution $\beta(\nu, \omega ; x)$ are:

$$
\mathrm{E}_{\mathrm{p}}(x)=-\operatorname{In}\left(\frac{x^{v-1}(1-x)^{\omega-1}}{\mathrm{~B}(v, \omega)}\right)=(1-v) \operatorname{In}(x)+(1-\omega) \operatorname{In}(1-x)+\operatorname{In}(\mathrm{B}(v, \omega)),
$$

where $\operatorname{In}(\mathrm{B}(v, \omega))$ can be considered as an additive constant that we can neglect, because it does not depend on the variable $\mathrm{x}$.

$$
\begin{gathered}
\mathrm{F}(x)=\frac{\partial \mathrm{E}_{\mathrm{p}}(x)}{\partial x}=\frac{(1-v)}{x}-\frac{(1-\omega)}{1-x}, \\
\mathrm{k}(x)=\frac{\partial^{2} \mathrm{E}_{\mathrm{p}}(x)}{\partial x^{2}}=-\frac{(1-v)}{x^{2}}-\frac{(1-\omega)}{(1-x)^{2}} .
\end{gathered}
$$

The mechanical properties linked to a lognormal distribution with the statistical quantities reported in Table 12, are shown in Table 19.

Table 12. Statistical properties of the probability distribution $\beta(10,2 ; x)$

\begin{tabular}{ll}
\hline$v$ & 10 \\
\hline$\omega$ & 2 \\
\hline mean & $v /(v+\omega) \simeq 0.83$ \\
\hline variance & $v \omega /\left[(v+\omega)^{2}(v+\omega+1)\right] \simeq 0.011$ \\
\hline mode & $(v-1) /(v+\omega-2)=0.9$ \\
\hline
\end{tabular}



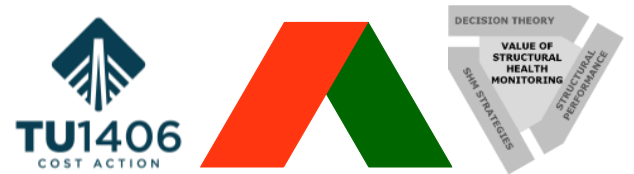

The Value of Structural Health Monitoring for the reliable Bridge Management

Zagreb 2-3 March 2017

\subsubsection{Trapezoidal distribution}

In probability theory and statistics, the trapezoidal distribution is a continuous probability distribution with lower limit $a$, upper limit $d$ and modes $b$ and $c$, where $a<d$ and $a \leq b \leq c \leq d$. Between $b$ and $c$, the probability density is constant; otherwise one has the generalized trapezoidal distribution. Special cases of the trapezoidal distribution include the uniform distribution (with $a=b$ and $c=d$ ) and the triangular distribution (with $b=c$ ). Trapezoidal distributions (Gao \& Sun) seem to be appropriate for modeling the duration and the form of a phenomenon which may be represented by three stages. The first stage can be viewed as a growth-stage, the second corresponds to a relative stability and the third represents a decline (decay). These distributions however are restricted since the growth and decay (in the first and third stages) are limited in the trapezoidal case to linear forms and the second stage represents complete stability rather than a possible mild incline or decline. The trapezoidal probability density function is of the form:

$$
\mathrm{f}(a, b, c, d ; x)=\left\{\begin{array}{c}
u \cdot\left(\frac{x-a}{b-a}\right) a \leq x<b \\
u \mathrm{~b} \leq x<c \quad, \quad \text { on }[a, d] \subset \mathbb{R} \\
u \cdot\left(\frac{d-x}{d-c}\right) \mathrm{c} \leq x<d
\end{array}\right.
$$

where $\mathrm{a}<\mathrm{b}<\mathrm{c}<\mathrm{d}$ and $u=\frac{2}{d+c-b-a}$.

The main statistic quantities of the trapezoidal distribution are reported in Table 13.

Table 13. Main statistical quantities of a random variable $x$, belonging to a trapezoidal distribution

\begin{tabular}{ll}
\hline mean & $(a+b+c+d) / 4$ \\
\hline variance & $\frac{(d-a)^{2}(1+\beta)^{2}}{24}$ \\
\hline mode & Any value in $[\mathrm{b}, \mathrm{c}]$ \\
\hline
\end{tabular}

where $0 \leq \beta<1$ represents the ratio between the smaller and the larger base of the trapezoidal function.

The potential, the elastic force and the stiffness function linked to the symmetric trapezoidal distribution $\mathrm{f}(a, b, c, d ; x)$ are:

$$
\begin{gathered}
\mathrm{E}_{\mathrm{p}}(x)=-\operatorname{In}(\mathrm{f}(a, b, c, d ; x))=\left\{\begin{array}{c}
-\operatorname{In}(u)+\operatorname{In}(b-a)-\operatorname{In}(x-a) a \leq x<b \\
-\operatorname{In}(\mathrm{u}) b \leq x<c \\
-\operatorname{In}(u)+\operatorname{In}(d-c)-\operatorname{In}(d-x) c \leq x<d
\end{array},\right. \\
\mathrm{F}(x)=\frac{\mathrm{dE}_{\mathrm{p}}(x)}{\mathrm{d} x}=\left\{\begin{array}{c}
-\frac{1}{x-a} a \leq x<b \\
0 b \leq x<c, \\
\frac{1}{d-x} c \leq x<d
\end{array}\right.
\end{gathered}
$$




$$
\mathrm{k}(x)=\frac{\mathrm{d}^{2} \mathrm{E}_{\mathrm{p}}(x)}{\mathrm{d} x^{2}}=\left\{\begin{array}{c}
\frac{1}{(x-a)^{2}} a \leq x<b \\
0 b \leq x<c \\
\frac{1}{(d-x)^{2}} c \leq x<d
\end{array} .\right.
$$

The mechanical properties linked to a lognormal distribution with the statistical quantities reported in Table 14, are shown in Table 19.

Table 14. Statistical properties of the probability distribution $\mathrm{f}(2,4,8,10 ; x)$

\begin{tabular}{ll}
\hline $\mathbf{a}$ & 2 \\
\hline $\mathbf{b}$ & 4 \\
\hline $\mathbf{c}$ & 8 \\
\hline $\mathbf{d}$ & 10 \\
\hline mean & $(a+b+c+d) / 4=6$ \\
\hline variance & $\frac{(d-a)^{2}(1+\beta)^{2}}{24}=6$ \\
\hline mode & $(a+b+c+d) / 4=6$ \\
\hline
\end{tabular}

\subsubsection{Triangular distribution}

In the theory of probability the triangular is a probability distribution continues whose probability density function describes a triangle (Forbes et al., 2011), or that it is nothing on the two extreme values and is linear between these and an intermediate value (the mode). In statistics is used as a model when the sample available is very limited. Its density probability function is the following:

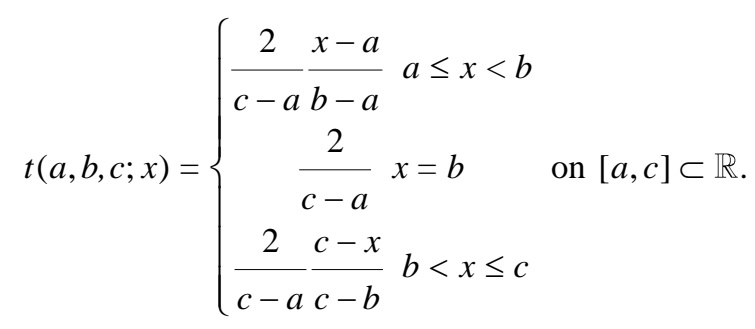

The main statistic quantities of the Cauchy distribution are reported in Table 15.

Table 15. Main statistical quantities of a random variable $\mathrm{x}$, belonging to a triangular distribution

\begin{tabular}{ll}
\hline mean & $(a+b+c) / 3$ \\
\hline variance & $\left(a^{2}+b^{2}+c^{2}-(a c+c b+a b)\right) / 18$ \\
\hline median & $a+\sqrt{(c-a)(b-a) / 2}$ se $b \geq \frac{a+c}{2}$ \\
\hline mode & $b$ \\
\hline
\end{tabular}

The potential, the elastic force and the stiffness function linked to the triangular distribution $\mathrm{t}(a, b, c ; x)$, defined on an interval $[a, c] \subset \mathbb{R}$ are: 


$$
\begin{aligned}
& \mathrm{E}_{\mathrm{p}}(x)=\left\{\begin{array}{rl}
-\operatorname{In}\left(\frac{2}{c-a} \frac{x-a}{b-a}\right) & =-\operatorname{In}(x-a)+\operatorname{In}(c-a)+\operatorname{In}(b-a)+k \quad a \leq x<b \\
-\operatorname{In}\left(\frac{2}{c-a}\right)=\operatorname{In}(c-a)+k \quad x=b & \\
-\operatorname{In}\left(\frac{2}{c-a} \frac{c-x}{c-b}\right) & =-\operatorname{In}(c-x)+\operatorname{In}(c-a)+\operatorname{In}(c-b)+k \quad b<x \leq c
\end{array},\right.
\end{aligned}
$$

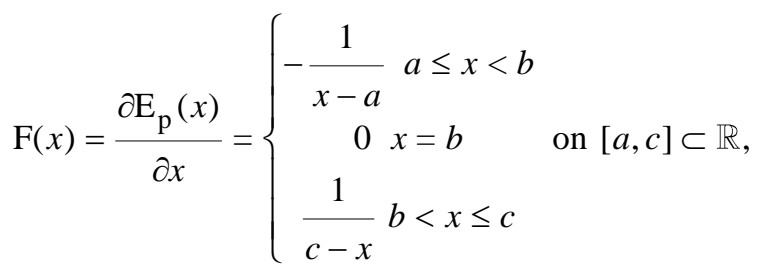

$$
\begin{aligned}
& \mathrm{k}(x)=\frac{\partial^{2} \mathrm{E}_{\mathrm{p}}(x)}{\partial x^{2}}=\left\{\begin{array}{cl}
\frac{1}{(x-a)^{2}} & a \leq x<b \\
0 & x=b \quad \text { on }[a, c] \subset \mathbb{R} . \\
\frac{1}{(c-x)^{2}} & b<x \leq c
\end{array}\right.
\end{aligned}
$$

The mechanical properties linked to a lognormal distribution with the statistical quantities reported in Table 16, are shown in Table 19.

Table 16. Statistical properties of the Probability distribution $\mathrm{t}(2,6,10 ; x)$

\begin{tabular}{ll}
\hline $\mathbf{a}$ & 2 \\
\hline $\mathbf{b}$ & 6 \\
\hline $\mathbf{c}$ & 10 \\
\hline mean & $(a+b+c) / 3=6$ \\
\hline variance & $\left(a^{2}+b^{2}+c^{2}-(a c+b c+a b)\right) / 18=2.67$ \\
\hline median & $a+\sqrt{(c-a)(b-a) / 2}=6 \quad$ se $b \geq \frac{a+c}{2}$ \\
\hline mode & $b=6$ \\
\hline
\end{tabular}

\subsubsection{Uniform distribution}

As we have seen before, if we analyzed a Beta distribution with the parameter that characterize it equal to one, $\beta(1,1 ; x)$, we obtain a uniform distribution (Forbes et al., 2011). In probability theory the uniform distribution is a probability distribution continues which is uniform on a set, or that attaches the same probability to all the points belonging to a given interval [a, b] contained in the set. The uniform distribution is usually defined on a continuous range $S=[a, b] \subset \mathbb{R}$; in this case it is indicated $U(a, b)=U([a, b])$. Its probability density is:

$$
u(a, b ; x)=\frac{1}{b-a} \text { on }[a, b] .
$$

The main statistic quantities of the Cauchy distribution are reported in Table 17. 
Table 17. Main statistical quantities of a random variable $\mathrm{x}$, belonging to a uniform distribution

\begin{tabular}{ll}
\hline mean & $(a+b) / 2$ \\
\hline variance & $\left(b^{2}-a^{2}\right) / 12$ \\
\hline median & $(a+b) / 2$ \\
\hline mode & any value in $[a, b] \subset \mathbb{R}$ \\
\hline
\end{tabular}

In this case it is not so difficult to understand that the potential of the associated spring will be constant, while the elastic force and the stiffness will result equal to zero in their entire domain.

$$
\begin{gathered}
\mathrm{E}_{\mathrm{p}}(x)=-\operatorname{In}\left(\frac{1}{b-a}\right)=\operatorname{In}(b-a), \\
\mathrm{F}(x)=\frac{\partial \mathrm{E}_{\mathrm{p}}(x)}{\partial x}=0, \\
\mathrm{k}(x)=\frac{\partial^{2} \mathrm{E}_{\mathrm{p}}(x)}{\partial x^{2}}=0 .
\end{gathered}
$$

The mechanical properties linked to a lognormal distribution with the statistical quantities reported in Table 18, are shown in Table 19.

Table 18. Statistical properties of the probability distribution $\mathrm{u}(2,10 ; x)$

\begin{tabular}{ll}
\hline $\mathbf{a}$ & 2 \\
\hline $\mathbf{b}$ & 10 \\
\hline mean & $(a+b) / 2=6$ \\
\hline variance & $\left(b^{2}-a^{2}\right) / 12=8$ \\
\hline median & $(a+b) / 2=6$ \\
\hline mode & any value in $[a, b] \subset \mathbb{R}$ \\
\hline
\end{tabular}

\subsubsection{Discussion of the results}

Table 19 shows the constitutive lows of the springs belonging to all the non-linear distributions presented in the previous sections. Particularly, it is interesting to note that the potential energy has a minimum in correspondence to the mode of the probability distribution and not in correspondence to the mean. In addition, the potential energy is not symmetric respect to its minimum value when the starting distribution is also not symmetric. In some cases, we notice that, when the displacements become remarkable, the elastic force becomes constant, by tending to a value little greater than zero, and in consequence the stiffness, which is the first derivative of the elastic force tends to zero.

\section{Conclusions}

We have stated an analogy between the world of logic and the world of mechanics, allowing us to solve, using the methods of classical structural engineering, any complex inference parameter estimation problem, in which the values of the parameters have to be estimated based on multiple Gaussian-distributed uncertain observations. By simply expressing the potential energy of the mechanical system associated to our inference scheme, we are able, with a few trivial algebraic steps, to determine the posterior mean values and standard deviations of the parameters to estimate. With the aid of real-life structural health monitoring cases, we have showed how our approach allows structural engineers to solve simply general problems of linear regression. Although the examples shown in this paper are incidentally all structural engineering cases, the scope of application of the method is 

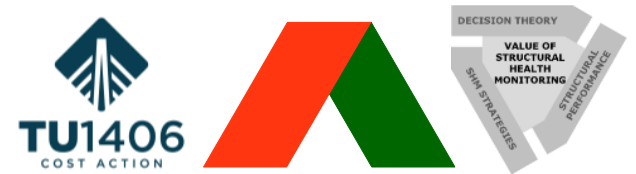

The Value of Structural Health Monitoring for the reliable Bridge Management

Zagreb 2-3 March 2017

evidently the most general, and we seek to demonstrate in the future its applicability to inference problem arising from various disciplinary fields, including cognitive science, economics and law.

\section{References}

D. Zonta, A. Lanaro, P. Zanon, "A strain-flexibility-based approach to damage location”, Key Engineering Materials (245-246), p.87-94, 2003.

D. Inaudi and B. Glisic, "Distributed fiber optic strain and temperature sensing for structural health monitoring", 3th IABMAS, 2006.

D. Zonta, H. Wu, M. Pozzi et al., "Wireless sensor networks for permanent health monitoring of historic buildings", Smart Structures and Systems 6 (5-6), p.595-618, 2010.

H. Sohn and K-H. Law, "Bayesian Probabilistic Damage Detection of a Reinforced Concrete Bridge Column", Earthquake Engineering and Structural Dynamics 29(8), 2000.

F. Bruschetta, D. Zonta, C. Cappello, et al., Bayesian analysis of monitoring data from cable-stayed bridge, 11th ICOSSAR, 2013.

E.T. Jaynes, Probability Theory: The Logic of Science, Cambridge: Cambridge University Press, 2003.

C. Cappello, D. Bolognani, D. Zonta, "Mechanical equivalent of logical inference from correlated uncertain information", Trento, 2015, in press.

H.H. Ku, "Notes on the Use of Propagation of Error Formulas", Journal of Research 70(4), 1966.

S.G. Rabinovich, Measurement Errors and Uncertainties: Theory and Practice, New York: Springer, 2005.

L. Kirkup and R.B. Frenkel, An Introduction to Uncertainty in Measurement, Cambridge: Cambridge University Press, 2006.

ISO5725-6:1994, International Organization for Standardization, 2012.

C.M. Bishop, 2006. Pattern Recognition and Machine Learning, Cambridge: Cambridge University Press.

M. Pozzi, D. Zonta, W. Wang et al., A framework for evaluating the impact of structural health monitoring on bridge management", 5th IABMAS, 2010.

C. Cappello, D. Zonta, M. Pozzi et al., "Impact of prior perception on bridge health diagnosis", Journal of Civil Structural health Monitoring (5), p. 509-525, 2015.

F. Bruschetta, D. Zonta, C. Cappello, et al., Fusion of monitoring data from cable-stayed bridge, 5th IEEE EESMS, 2013.

B. Glišić, D. Posenato and D. Inaudi, D., Integrity monitoring of old steel bridge using fiber optic distributed sensors based on Brillouin scattering, Proceedings of SPIE, 2007.

D. Zonta, A. Chappini, A. Chiasera, et al., Photonic crystals for monitoring fatigue phenomena in steel structures, Proceedings of SPIE, 2009.

J. P. Ward, "Solid Mechanics: an introduction”, Kluwer Academic, 1992.

C. Forbes et al., Statistical distributions, New Jersey, 2011.

X. Gao and S. Sun, Variance Formula for Trapezoidal Uncertain Variables, North China Electric Power University, China. 
Table 19. Constitutive lows of the springs belonging to different kinds of non-linear distributions

\begin{tabular}{|c|c|c|c|}
\hline & Gaussian & Lognormal & Gumbel \\
\hline $\begin{array}{c}\text { Distribution } \\
\qquad f(x)\end{array}$ & 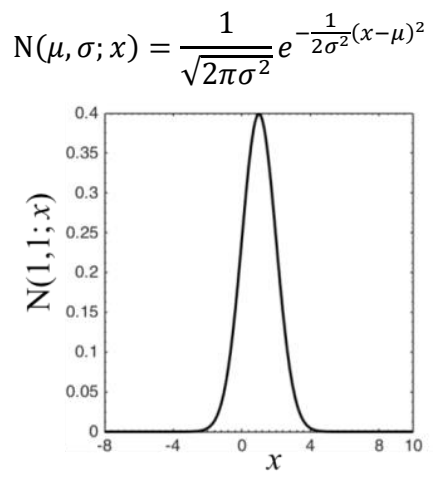 & $l(\lambda, \varepsilon ; x)=\frac{1}{x \varepsilon \sqrt{2 \pi}} e^{-\frac{1}{2 \varepsilon^{2}}(I n(x)-\lambda)^{2}}$ & \begin{tabular}{l}
$g(a, b ; x)=\frac{1}{b} e^{-\frac{-(x-a)}{b}-e^{\frac{-(x-a)}{b}}}$ \\
\hdashline
\end{tabular} \\
\hline $\begin{array}{c}\begin{array}{c}\text { Potential } \\
\text { energy }\end{array} \\
\mathrm{E}_{p}(x)= \\
-I n(f(x))\end{array}$ & $\mathrm{E}_{p}(x)=\frac{1}{2} w(x-\mu)^{2}$ & 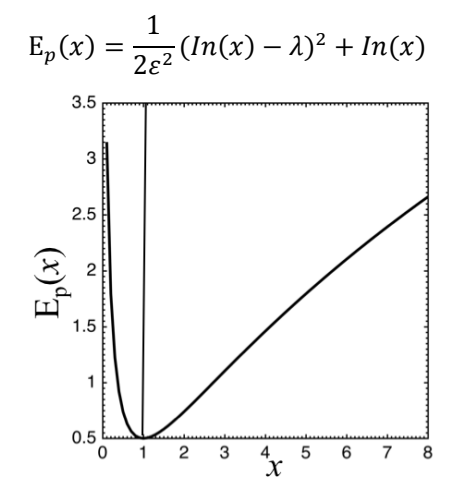 & 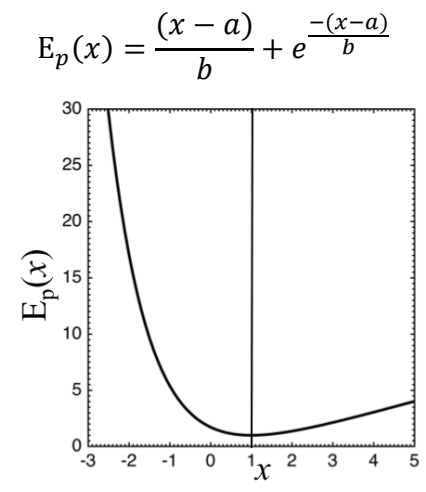 \\
\hline $\begin{array}{l}\begin{array}{l}\text { Elastic } \\
\text { force }\end{array} \\
\mathrm{F}(x)= \\
\frac{d \mathrm{E}_{p}(x)}{d x}\end{array}$ & $\int_{1}^{4} x^{7}$ & 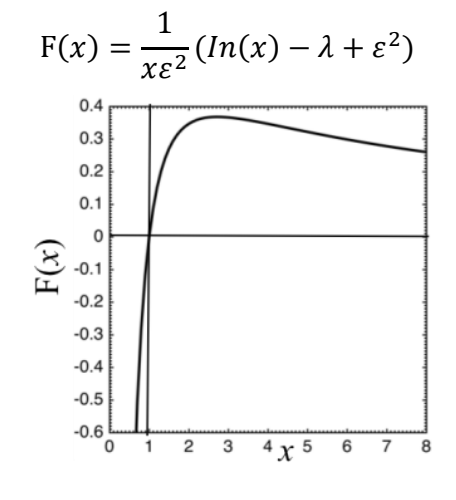 & 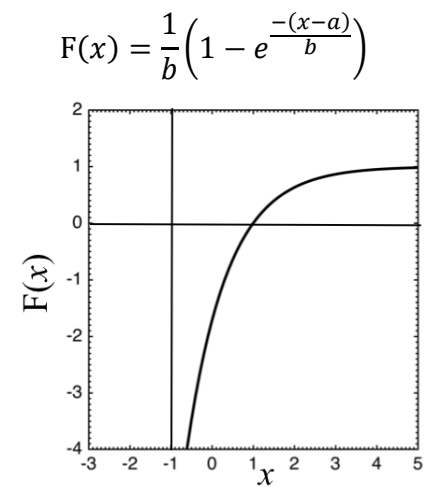 \\
\hline $\begin{array}{l}\text { Stiffness } \\
k(x)= \\
\frac{\mathrm{d}^{2} \mathrm{E}_{\mathrm{p}}(\mathrm{x})}{\mathrm{dx}^{2}}\end{array}$ & 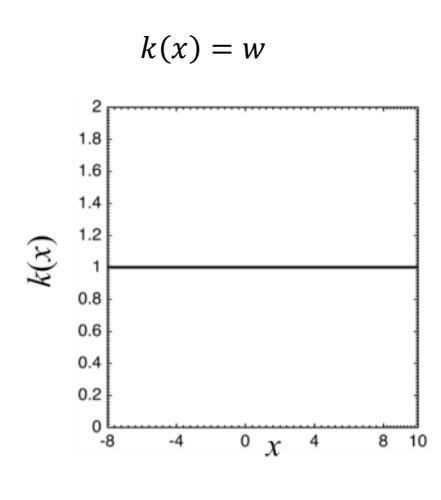 & 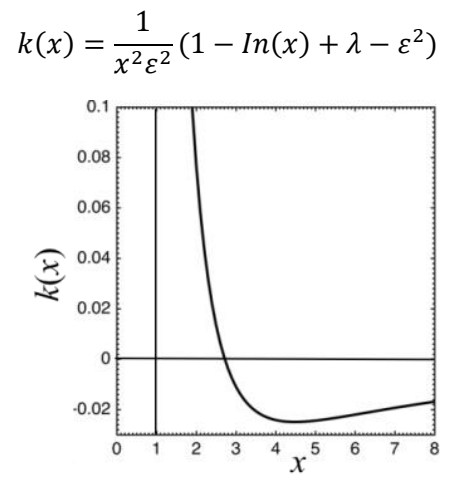 & 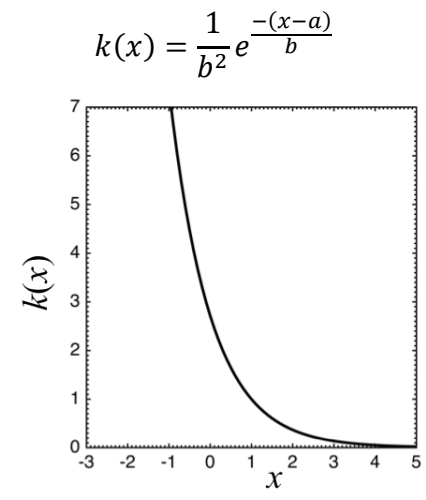 \\
\hline
\end{tabular}




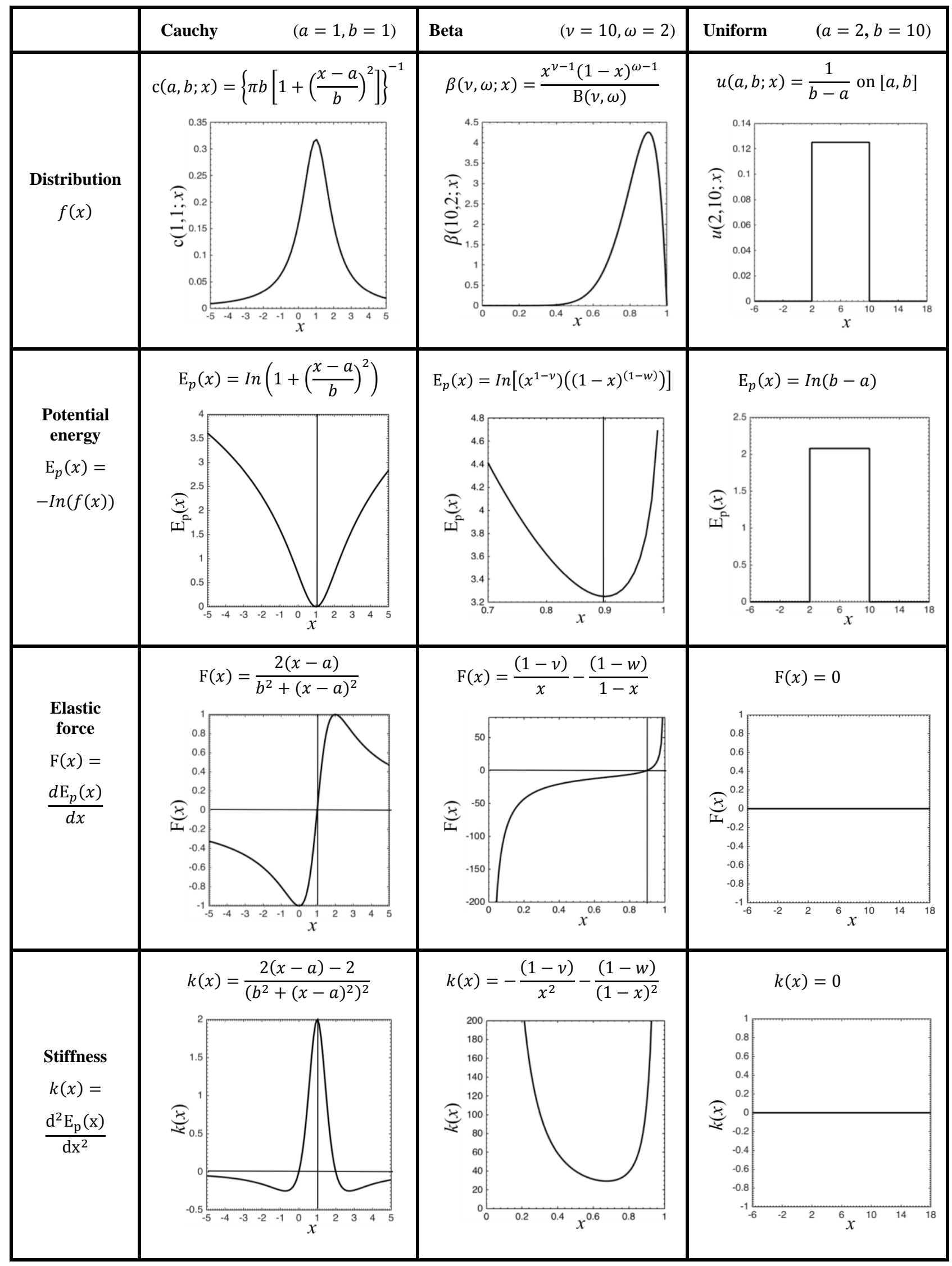




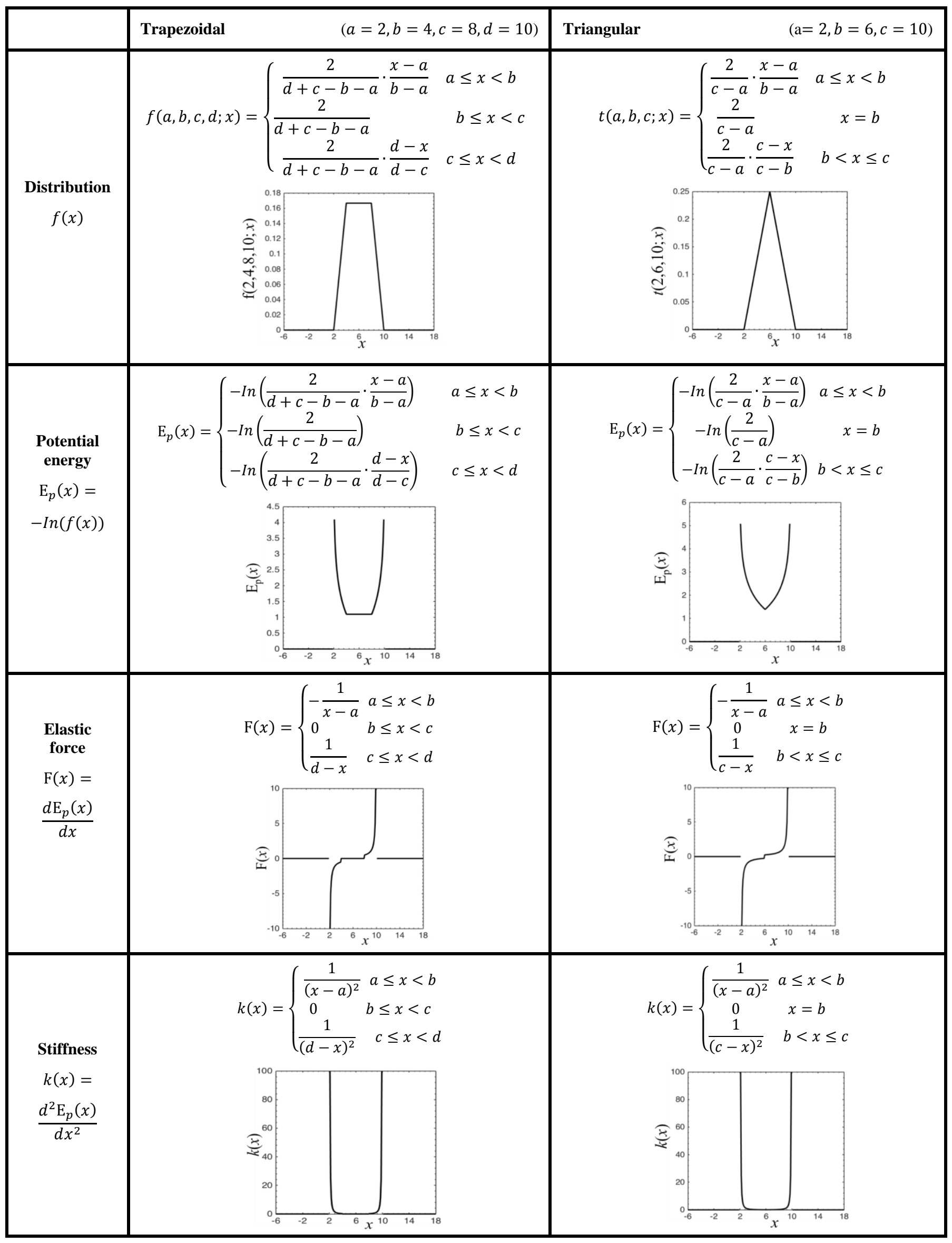

